Tropical Journal of Pharmaceutical Research January 2019; 18 (1): 193-201

ISSN: $1596-5996$ (print); 1596-9827 (electronic)

(C) Pharmacotherapy Group, Faculty of Pharmacy, University of Benin, Benin City, 300001 Nigeria.

\title{
Effectiveness and safety of Compound Danshen injection as treatment for pregnancy-induced hypertension: A meta- analysis
}

\author{
Xiuqin Wang ${ }^{1}$, Donghua $\mathrm{Guo}^{2}$, Ailong Yang ${ }^{3}$, Yu Wang ${ }^{3}$, Ruifang Wang ${ }^{4}$, Jiale \\ $\mathbf{L i}^{1 *}$ \\ ${ }^{1}$ Department of Pharmacy, ${ }^{2}$ Blood Purification Center, ${ }^{3}$ Medical department, ${ }^{4}$ Nursing Department, 263 Clinical Departments of \\ the Army General Hospital, Beijing, China
}

*For correspondence: Email: wangxqbj@21cn.com

Sent for review: 18 September 2018

Revised accepted: 11 December 2018

\begin{abstract}
Purpose: To systematically evaluate the effectiveness and safety of Compound Danshen injection in patients with pregnancy-induced hypertension (PIH).

Methods: PubMed, Embase, Cochrane Library, Chinese biomedical literature database (CBM), VIP (xiAn), China National Knowledge Infrastructure (CNKI) and Wan Fang databases were searched up to March 20, 2018, for all randomized controlled trials (RCTs) on the use of Compound Danshen injection in patients with PIH. Data were extracted from included studies after assessing the quality of literature. Revman 5.3 software was used for statistical analysis.

Results: A total of 18 RCTs involving 1735 patients were included. The results of meta-analysis indicated that the study group was superior to the control group in clinical effectiveness ( $R R=1.15,95$ $\% \mathrm{Cl}: 1.02-1.30$ ); intrauterine fetal distress ( $R R=0.26,95 \% \mathrm{Cl}: 0.09-0.70)$; cesarean section (RR = $0.72,95 \% \mathrm{Cl}: 0.58-0.90)$, and neonatal asphyxia $(R R=0.23,95 \% \mathrm{Cl}: 0.11-0.48)$. There were no statistical differences in fetal heart rate abnormalities $(R R=0.58,95 \%, C l: 0.33-1.02, p>0.05)$ and postpartum hemorrhage (RR=0.86, $95 \% \mathrm{Cl}: 0.53$ - 1.42) between the two groups.

Conclusion: Treatment of PIH with Compound Danshen injection (alone or in combination) is superior to the use of conventional western medical treatment in safety and effectiveness. However, higher quality clinical studies are needed to confirm these results because most trials included in this study were of low quality.
\end{abstract}

Keywords: Pregnancy-induced hypertension (PIH), Compound Danshen injection, Meta-analysis

This is an Open Access article that uses a funding model which does not charge readers or their institutions for access and distributed under the terms of the Creative Commons Attribution License (http://creativecommons.org/licenses/by/4.0) and the Budapest Open Access Initiative (http://www.budapestopenaccessinitiative.org/read), which permit unrestricted use, distribution, and reproduction in any medium, provided the original work is properly credited.

Tropical Journal of Pharmaceutical Research is indexed by Science Citation Index (SciSearch), Scopus, International Pharmaceutical Abstract, Chemical Abstracts, Embase, Index Copernicus, EBSCO, African Index Medicus, JournalSeek, Journal Citation Reports/Science Edition, Directory of Open Access Journals (DOAJ), African Journal Online, Bioline International, Open-J-Gate and Pharmacy Abstracts

\section{INTRODUCTION}

Pregnancy induced hypertension $(\mathrm{PIH})$ is a condition in pregnant women with elevated systolic $(\geq 140 \mathrm{mmHg})$ or diastolic $(\geq 90 \mathrm{mmHg})$ blood pressure on at least two occasions $6 \mathrm{~h}$ apart. Generally, it occurs after 20 weeks of gestation and returns to normal 12 weeks postpartum. It is the most common obstetrical complication in pregnancy, and a leading cause of maternal and perinatal mortality and morbidity. Pregnancy induced hypertension $(\mathrm{PIH})$ is 
characterized by hypertension, proteinuria and edema.

Compound Danshen injection is extracted from the dry roots of Salvia miltiorrhiza and dalbergiae. Salvia miltiorrhiza and dalbergiae exert vasodilation effects and improve microcirculation. At present, there are no systemic evaluations on the use of Compound Danshen for treatment of $\mathrm{PIH}$. This study was based on the results of related clinical studies on the treatment of PIH with Compound Danshen injection. It was aimed at investigating the clinical effectiveness and safety of this injection so as to provide evidence-based data in support of its application as therapy for PIH.

\section{METHODS}

\section{Data sources}

Studies published in English and Chinese on the use of Compound Danshen injection for $\mathrm{PIH}$ treatment were searched using PubMed, Embase, Cochrane Library, CBM, VIP (XiAn), $\mathrm{CNKI}$ and Wan Fang databases as the main sources. The internet sources were supplemented with relevant documents by searching references cited in the studies, and manually searching other relevant documents to obtain additional information. The search covered all previous studies up to March 20, 2018. The Chinese terms used for searching were "Compound Danshen", "pregnancy with hypertension", "rengaozheng", while the English terms used were "Pregnancy-induced hypertension", "Danshen" and "PIH". Taking PubMed and CNKI as examples, the key PubMed search words/word combinations were: pregnancy-induced hypertension, Danshen, Chinese herbal medicine, Chinese traditional medicine herb, clinical observation, as well as combinations of these search words. The CNKI key search words/word combinations were: "hypertension during pregnancy", "pregnancyinduced hypertension", Salvia miltiorrhizae, clinical research, and combinations of these words.

\section{Inclusion criteria}

The included studies met the following criteria: studies describing results from intervention measures in randomized controlled trials (RCT) of Compound Danshen injection in the treatment of $\mathrm{PIH}$, using blinded or unblinded methods; studies with clear clinical diagnosis of PIH in pregnant women; studies in which the research indicators included total effectiveness, fetal heart rate abnormalities, incidence of intrauterine fetal distress, frequency of caesarean section, incidence of postpartum hemorrhage, frequency of occurrence of neonatal asphyxia, and other adverse outcomes; and studies in which the observation group was treated with Compound Danshen injection or with Compound Danshen injection combined with conventional Western medicine, while the control group received conventional Western medicine alone.

\section{Exclusion criteria}

The following studies were excluded: nonrandomized controlled trials or quasi-randomized controlled trials (animal experimental studies, theoretical discussions, review and experience summaries, case reports, and analytical articles); studies with small data sets (these were excluded due to duplicated publications); and studies from which literature on the required outcomes could not be extracted.

\section{Data extraction}

The following information were extracted from each publication: name of first author, year of publication, location, age, gestational weeks, parity, sample size, clinical effectiveness and assessment of maternal and child outcomes in both observation group and control groups. All information were checked and collected independently by two researchers, and any inconsistencies were examined and discussed until a unanimous interpretation was reached. The citations were arranged by year of publication in tables.

\section{Study quality assessment}

According to the JADAD rating scale for quality assessment, this study was based on the following elements: random sequence generation (selection bias), allocation concealment (selection bias), blinding (implementation bias), and withdrawal and drop out. The literature was scored according to specific content. The JADAD assigns a maximum score of 0 - 2 for the first three factors, and $0-1$ for the last factor. Usually, studies with JADAD score of 3 or higher are regarded as high-quality, whereas those with JADAD scores less than 2 are considered lowquality studies. In this study, if the random grouping sequence was generated by computerassisted random sequence or random number table, the study was scored 2 points; if the experiment mentioned random allocation, but the method of generating random sequence was not indicated, it was scored 1 point, while semirandom or quasi-random randomized trials referring to the method of alternating cases such 
as the order of admission and the number of single births were scored 0 point.

\section{Statistical analysis}

RevMan 5.3 software was used for statistical analysis. Enumeration data was analyzed using relative risk (RR) and $95 \%$ confidence intervals (Cls). Measurement data was analyzed using mean difference (MD) and $95 \%$ confidence intervals (Cls). Values of $p<0.05$ were considered statistically significant. Combined odds ratios (RRs) and $95 \%$ confidence intervals (Cls) were calculated using RevMan software, with a forest plot showing the characteristics of the various findings. When $p>0.1$ and $\mathrm{I}^{2}<50 \%$, a fixed-effect model was used for meta-analysis; but when $p<0.1$ and $I^{2}>50 \%$, a random-effect model was used for meta-analysis. The standard errors of the RR natural logarithm were taken as abscissa and the RR natural logarithm were used for the vertical axis to draw a funnel plot used in the determination of publication bias.

\section{RESULTS}

\section{Characteristics of included studies}

As shown in Table 1, a total of 89 publications were selected after the final search. After excluding duplicated research and unrelated studies, 18 studies (numbered $1-18$ in superscript) involving 1735 subjects were eventually included in the present study. Nine (09) out of the 18 studies $(6,7,9,10,14-18)$ showed total effectiveness values; 03 (9, 13 18) described fetal heart abnormalities; 03 (11, 14,
16) involved intrauterine fetal distress; 04 (9. 11, $13,18)$ discussed cesarean section; 05 (9, 11, $14,16,18)$ reported postpartum hemorrhage, 05 $(9,11,13,14,18)$ mentioned neonatal asphyxia, while $03(1,2,7)$ reported adverse reactions in observation groups and control groups.

\section{Results of evaluation of literature quality (JADAD)}

Eighteen (18) articles involving 876 patients and 859 controls met the selection criteria. The lowest sample size was 24, and highest sample size was 177 . The present study included a total of 18 RCTs. Meta-analysis showed that the studies were of low quality. The characteristics of the included studies and treatments used against $\mathrm{PIH}$ are shown in Table 1, Table 2 and Table 3.

\section{Meta-analysis results}

\section{Total effectiveness}

Nine (09) studies showed total overall effectiveness. Heterogeneity test revealed significant heterogeneity among the various studies $\left(p<0.00001, \mathrm{I}^{2}=80 \%\right)$. Thus, randomeffect model was used to calculate RRs. Metaanalysis showed that total effectiveness differed significantly between the two groups $(R R=1.15$, $95 \% \mathrm{Cl}: 1.02-1.30, p=0.02)$. These results are shown in Figure 1, Figure 2 and Figure 3. The total effectiveness of Compound Danshen injection alone or in combination with Western medicine in the treatment of $\mathrm{PIH}$ was higher than that of Western medicine alone.

Table 1: Basic information on included studies

\begin{tabular}{|c|c|c|c|c|c|c|}
\hline \multirow{2}{*}{$\begin{array}{c}\text { Authors } \\
\text { (year) }\end{array}$} & \multirow{2}{*}{$\begin{array}{l}\text { N(control } \\
\text { /study) }\end{array}$} & \multirow{2}{*}{$\begin{array}{l}\text { Mean age, age } \\
\text { (control/study) }\end{array}$} & \multicolumn{2}{|c|}{ Intervention measures } & \multirow{2}{*}{$\begin{array}{l}\text { Pregnancy, week } \\
\text { (control/study) }\end{array}$} & \multirow{2}{*}{$\begin{array}{l}\text { Measurement } \\
\text { index }\end{array}$} \\
\hline & & & Control & Study & & \\
\hline $\begin{array}{l}\operatorname{Ren}^{[1]} \\
(2004)\end{array}$ & $40 / 40$ & $25 \sim 36$ & $\begin{array}{l}\text { Magnesium sulfate } \\
+ \text { nifedipine }\end{array}$ & Compound Danshen & $27 \sim 40$ & Clinical effectiveness \\
\hline $\begin{array}{l}\mathrm{Ma}^{\mathrm{l}^{2 /}} \\
(2008)\end{array}$ & $30 / 30$ & No & $\begin{array}{l}\text { Western } \\
\text { medicine }\end{array}$ & $\begin{aligned} & \text { Western medicine } \\
+ & \text { Compound Danshen }\end{aligned}$ & Not mentioned & Clinical effectiveness \\
\hline $\begin{array}{l}\text { Guo }^{[3]} \\
(2009)\end{array}$ & $23 / 27$ & $22 \sim 35$ & $\begin{array}{l}\text { Western } \\
\text { medicine }\end{array}$ & $\begin{aligned} & \text { Western medicine } \\
&+ \text { Compound Danshen }\end{aligned}$ & $28 \sim 38$ & Clinical effectiveness \\
\hline $\begin{array}{l}\mathrm{Hao}^{[4]} \\
(2009)\end{array}$ & $28 / 28$ & $\begin{array}{l}27.4 \pm 3.1 \\
/ 27.8 \pm 3.2\end{array}$ & Magnesium sulfate & $\begin{array}{l}\text { Magnesium Sulfate } \\
+ \text { Compound Danshen }\end{array}$ & $\begin{array}{l}37.0 \pm 1.9 \\
/ 36.5 \pm 2.4\end{array}$ & Clinical effectiveness \\
\hline $\begin{array}{l}\mathrm{CHu}^{[5]} \\
(2012)\end{array}$ & $24 / 24$ & $\begin{array}{l}27.4 \pm 2.6 \\
/ 27.8 \pm 1.9\end{array}$ & Magnesium sulfate & $\begin{aligned} & \text { Magnesium Sulfate } \\
+ & \text { Compound Danshen }\end{aligned}$ & $\begin{array}{l}36.7 \pm 1.4 \\
/ 35.8 \pm 1.1\end{array}$ & Clinical effectiveness \\
\hline $\begin{array}{l}\text { ZHeng }^{[6]} \\
(2013)\end{array}$ & $37 / 40$ & $26.9 \pm 3.3$ & Magnesium sulfate & $\begin{array}{l}\text { Magnesium Sulfate } \\
+ \text { Compound Danshen }\end{array}$ & $32.7 \pm 1.8$ & Clinical effectiveness \\
\hline $\begin{array}{l}\mathrm{Jia}^{\prime\rfloor} \\
\text { (2013) }\end{array}$ & $80 / 80$ & $34 / 35$ & Magnesium sulfate & $\begin{aligned} & \text { Magnesium Sulfate } \\
+ & \text { Compound Danshen }\end{aligned}$ & $33 / 34$ & Clinical effectiveness \\
\hline $\begin{array}{r}\text { Wang } \\
(2014)\end{array}$ & $50 / 50$ & $28.1 \pm 2.3$ & $\begin{array}{l}\text { Western } \\
\text { medicine }\end{array}$ & $\begin{aligned} & \text { Western medicine } \\
+ & \text { Compound Danshen }\end{aligned}$ & $35.7 \pm 3.1$ & Clinical effectiveness \\
\hline $\begin{array}{l}\text { Wen }^{[9]} \\
(2014)\end{array}$ & $30 / 30$ & $\begin{array}{l}28.2 \pm 2.7 \\
/ 29.3 \pm 2.6\end{array}$ & $\begin{array}{l}\text { Western } \\
\text { medicine }\end{array}$ & $\begin{aligned} & \text { Western medicine } \\
+ & \text { Compound Danshen }\end{aligned}$ & $\begin{array}{l}33.5 \pm 0.8 \\
/ 32.7 \pm 1.2\end{array}$ & $\begin{array}{l}\text { Clinical effectiveness } \\
\text { Maternal and infant } \\
\text { pregnancy Outcome }\end{array}$ \\
\hline
\end{tabular}


Table 2: Basic information on the included studies

\begin{tabular}{|c|c|c|c|c|c|c|}
\hline \multirow[b]{2}{*}{$\begin{array}{l}\text { Authors } \\
\text { (year) }\end{array}$} & \multirow[b]{2}{*}{$\begin{array}{l}\mathrm{N} \text { (control } \\
\text { /study) }\end{array}$} & \multirow[b]{2}{*}{$\begin{array}{l}\text { Average age, age } \\
\text { (control/study) }\end{array}$} & \multicolumn{2}{|c|}{ Intervention measures } & \multirow{2}{*}{$\begin{array}{l}\text { Pregnancy, } \\
\text { week } \\
\text { (control / } \\
\text { study) }\end{array}$} & \multirow[b]{2}{*}{ Measurement index } \\
\hline & & & Control & Study & & \\
\hline $\begin{array}{l}\text { ZHang[10] } \\
(2015)\end{array}$ & $176 / 177$ & $\begin{array}{l}27.9 \pm 1.6 \\
/ 28.3 \pm 1.4\end{array}$ & $\begin{array}{l}\text { Western } \\
\text { medicine }\end{array}$ & $\begin{array}{l}\text { Western medicine } \\
+ \text { Compound Danshen }\end{array}$ & $\begin{array}{l}38.0 \pm 1.5 \\
/ 32.7 \pm 1.4\end{array}$ & Clinical effectiveness \\
\hline $\begin{array}{l}\text { CHen[11] } \\
(2015)\end{array}$ & $60 / 60$ & $\begin{array}{l}27.0 \pm 3.0 \\
/ 27.0 \pm 3.2\end{array}$ & $\begin{array}{l}\text { Western } \\
\text { medicine }\end{array}$ & $\begin{array}{l}\text { Western medicine } \\
\text { + Compound Danshen }\end{array}$ & $\begin{array}{l}36.0 \pm 0.9 \\
/ 36.2 \pm 0.8\end{array}$ & $\begin{array}{l}\text { Clinical effectiveness } \\
\text { Maternal and infant } \\
\text { pregnancy Outcome }\end{array}$ \\
\hline $\begin{array}{l}\text { Qiu[12] } \\
\text { (2016) }\end{array}$ & $45 / 54$ & $\begin{array}{l}27.8 \pm 1.3 \\
/ 26.1 \pm 1.4\end{array}$ & $\begin{array}{l}\text { Magnesium } \\
\text { Sulfate }\end{array}$ & $\begin{array}{l}\text { Magnesium Sulfate } \\
\text { + Compound Danshen }\end{array}$ & $\begin{array}{l}37.73 \pm 4.33 \\
/ 36.42 \pm 5.12\end{array}$ & Clinical effectiveness \\
\hline $\begin{array}{l}\mathrm{Ii}[13] \\
\quad(2016)\end{array}$ & $45 / 45$ & $\begin{array}{l}29.5 \pm 4.6 \\
/ 28.5 \pm 4.9\end{array}$ & $\begin{array}{l}\text { Western } \\
\text { medicine }\end{array}$ & $\begin{array}{l}\text { Western medicine } \\
+ \text { Compound Danshen }\end{array}$ & $\begin{array}{l}36.6 \pm 2.58 \\
/ 36.2 \pm 2.48\end{array}$ & $\begin{array}{l}\text { Clinical effectiveness } \\
\text { Maternal and infant } \\
\text { pregnancy Outcome }\end{array}$ \\
\hline $\begin{array}{l}\text { Wang[14] } \\
(2016)\end{array}$ & $60 / 60$ & $\begin{array}{l}26.7 \pm 5.3 \\
/ 27.5 \pm 4.5\end{array}$ & $\begin{array}{l}\text { Western } \\
\text { medicine }\end{array}$ & $\begin{array}{l}\text { Western medicine } \\
\text { + Compound Danshen }\end{array}$ & $\begin{array}{l}34.3 \pm 2.7 \\
/ 35.2 \pm 2.8\end{array}$ & $\begin{array}{l}\text { Clinical effectiveness } \\
\text { Maternal and infant } \\
\text { pregnancy outcome }\end{array}$ \\
\hline $\begin{array}{l}\text { Ding[15] } \\
(2016)\end{array}$ & $30 / 30$ & $24.9 \pm 2.9 / 25.9 \pm 2.8$ & $\begin{array}{l}\text { Western } \\
\text { medicine }\end{array}$ & $\begin{array}{l}\text { Western medicine } \\
+ \text { Compound Danshen }\end{array}$ & Not mentioned & Clinical effectiveness \\
\hline $\begin{array}{l}\text { ZHang[16] } \\
(2016)\end{array}$ & $39 / 39$ & $\begin{array}{l}26.83 \pm 2.12 \\
/ 27.21 \pm 2.37\end{array}$ & Nifedipine & $\begin{array}{l}\text { Nifedipine } \\
\text { + Compound Danshen }\end{array}$ & $\begin{array}{l}33.24 \pm 1.46 \\
/ 32.91 \pm 0.85\end{array}$ & Clinical effectiveness \\
\hline $\begin{array}{l}\text { ZHao[17] } \\
(2017)\end{array}$ & $30 / 30$ & $24.6 \pm 2.2$ & $\begin{array}{l}\text { Magnesium } \\
\text { Sulfate }\end{array}$ & $\begin{array}{l}\text { Magnesium Sulfate } \\
\text { + Compound Danshen }\end{array}$ & Not mentioned & Clinical effectiveness \\
\hline $\begin{array}{l}\text { ZHang[18] } \\
(2017)\end{array}$ & $32 / 32$ & $\begin{array}{l}26.7 \pm 3.3 \\
/ 27.8 \pm 2.2\end{array}$ & $\begin{array}{l}\text { Western } \\
\text { medicine }\end{array}$ & $\begin{array}{l}\text { Western medicine } \\
\text { + Compound Danshen }\end{array}$ & $\begin{array}{l}35.2 \pm 2.5 \\
/ 34.8 \pm 3.3\end{array}$ & $\begin{array}{l}\text { Clinical effectiveness } \\
\text { Maternal and infant } \\
\text { pregnancy outcome }\end{array}$ \\
\hline
\end{tabular}

Table 3: Characteristics of included studies in the study sample

\begin{tabular}{|c|c|c|c|c|}
\hline $\begin{array}{l}\text { Authors } \\
\text { (year)* }^{\star}\end{array}$ & $\begin{array}{l}\text { n (control } \\
\text { /study) }\end{array}$ & Random method & JADAD score & Measurement index \\
\hline $\operatorname{Ren}^{[1]}(2004)$ & $40 / 40$ & RCT & 1 & Clinical effectiveness \\
\hline $\mathrm{Ma}^{[2]}(2008)$ & $30 / 30$ & $\mathrm{RCT}$ & 1 & Clinical effectiveness \\
\hline $\mathrm{Guo}^{[3]}(2009)$ & $23 / 27$ & $\mathrm{RCT}$ & 1 & Clinical effectiveness \\
\hline $\mathrm{Hao}^{[4]}(2009)$ & $28 / 28$ & RCT & 1 & Clinical effectiveness \\
\hline $\mathrm{CHu}^{[5]}(2012)$ & $24 / 24$ & RCT & 1 & Clinical effectiveness \\
\hline ZHeng $^{[6]}$ (2013) & $37 / 40$ & $\mathrm{RCT}$ & 1 & Clinical effectiveness \\
\hline $\mathrm{Jia}^{[7]}(2013)$ & $80 / 80$ & RCT & 1 & Clinical effectiveness \\
\hline Wang $^{[8]}$ (2014) & $50 / 50$ & RCT & 1 & Clinical effectiveness \\
\hline Wen $^{[9]}(2014)$ & $30 / 30$ & $\mathrm{RCT}$ & 2 & $\begin{array}{l}\text { Clinical effectiveness } \\
\text { Maternal and infant pregnancy outcome }\end{array}$ \\
\hline ZHang $^{[10]}$ (2015) & $176 / 177$ & $\mathrm{RCT}$ & 2 & $\begin{array}{l}\text { Clinical effectiveness } \\
\text { Clinical effectiveness }\end{array}$ \\
\hline $\mathrm{CHen}^{[11]}$ (2015) & $60 / 60$ & $\mathrm{RCT}$ & 2 & $\begin{array}{l}\text { Maternal and infant pregnancy } \\
\text { Outcome }\end{array}$ \\
\hline Qiu $^{[12]}$ (2016) & $45 / 54$ & RCT & 2 & Clinical effectiveness \\
\hline $\mathrm{li}^{[13]}(2016)$ & $45 / 45$ & $\mathrm{RCT}$ & 2 & $\begin{array}{l}\text { Clinical effectiveness } \\
\text { Maternal and infant pregnancy outcome } \\
\text { Clinical effectiveness }\end{array}$ \\
\hline Wang $^{[14]}(2016)$ & $60 / 60$ & $\mathrm{RCT}$ & 2 & $\begin{array}{l}\text { maternal and infant pregnancy } \\
\text { Outcome }\end{array}$ \\
\hline $\operatorname{Ding}^{[15]}(2016)$ & $30 / 30$ & RCT & 1 & Clinical effectiveness \\
\hline ZHang $^{[16]}$ (2016) & $39 / 39$ & $\mathrm{RCT}$ & 1 & Clinical effectiveness \\
\hline $\mathrm{ZHao}^{[17]}$ (2017) & $30 / 30$ & $\mathrm{RCT}$ & 1 & Clinical effectiveness \\
\hline ZHang $^{[18]}$ (2017) & $32 / 32$ & $\mathrm{RCT}$ & 1 & $\begin{array}{l}\text { Clinical effectiveness } \\
\text { maternal and infant pregnancy outcome }\end{array}$ \\
\hline
\end{tabular}

${ }^{*}$ Superscripts refer to serial numbers of authors/publications 


\begin{tabular}{|c|c|c|c|c|c|c|c|}
\hline \multirow[b]{2}{*}{ Study or Subgroup } & \multicolumn{2}{|c|}{ Observation } & \multicolumn{2}{|c|}{ Control } & \multirow{2}{*}{ Weight } & \multirow{2}{*}{$\begin{array}{c}\text { Risk Ratio } \\
\text { M-H. Random, } 95 \% \mathrm{Cl}\end{array}$} & \multirow{2}{*}{$\begin{array}{c}\text { Risk Ratio } \\
\mathrm{M}-\mathrm{H}, \text { Random. } 95 \% \mathrm{Cl}\end{array}$} \\
\hline & Events & Total & Events & Total & & & \\
\hline Ding2016 & 29 & 30 & 23 & 30 & $10.0 \%$ & $1.26[1.02,1.55]$ & + \\
\hline $\mathrm{Jia} 2013$ & 60 & 80 & 76 & 80 & $12.2 \%$ & $0.79[0.69,0.90]$ & - \\
\hline Wang2016 & 58 & 60 & 50 & 60 & $12.6 \%$ & $1.16[1.03,1.31]$ & - \\
\hline Wen2014 & 27 & 30 & 22 & 30 & $8.9 \%$ & $1.23[0.96,1.57]$ & - \\
\hline Zhang2015 & 165 & 177 & 124 & 176 & $13.1 \%$ & $1.32[1.19,1.47]$ & - \\
\hline Zhang2016 & 37 & 39 & 28 & 39 & $10.0 \%$ & $1.32[1.07,1.63]$ & - \\
\hline Zhang2017 & 31 & 32 & 27 & 32 & $11.5 \%$ & $1.15[0.98,1.35]$ & $=$ \\
\hline Zhao2017 & 27 & 30 & 22 & 30 & $8.9 \%$ & $1.23[0.96,1.57]$ & $F$ \\
\hline Zheng2013 & 39 & 40 & 33 & 37 & $12.6 \%$ & $1.09[0.97,1.24]$ & $=$ \\
\hline Total $(95 \% \mathrm{Cl})$ & & 518 & & 514 & $100.0 \%$ & $1.15[1.02,1.30]$ & 1 \\
\hline Total events & 473 & & 405 & & & & \\
\hline
\end{tabular}

Figure 1: Forest plot of meta-analysis of total effectiveness

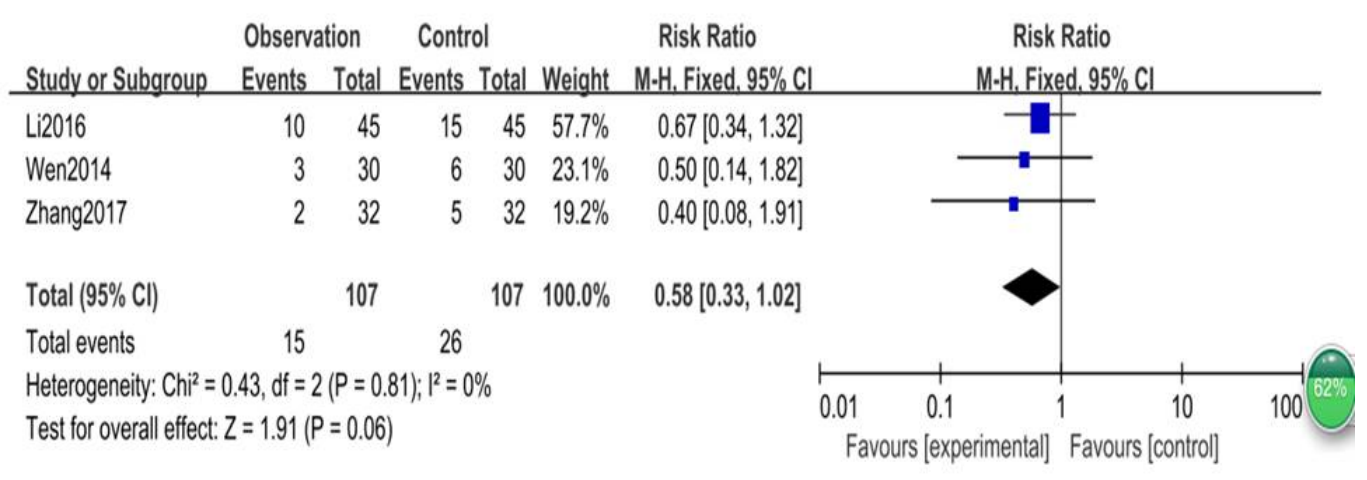

Figure 2: Forest plot of meta-analysis of fetal heart abnormalities

\section{Fetal heart abnormalities}

Three (03) studies described fetal heart abnormalities, but results of the statistics were not heterogeneous ( $p=0.81, \mathrm{I}^{2}=0 \%$, Figure 2). Therefore, fixed-effect model was used to calculate RRs. Meta-analysis showed absence of statistically significant differences in fetal heart abnormalities between the two groups ( $R R=$ 0.58, $95 \% \mathrm{Cl}: 0.33-1.02, p>0.05)$.

\section{Intrauterine fetal distress}

Three (03) studies dwelt on intrauterine fetal distress. The results of statistical analysis did not show heterogeneity in the three studies $(p=$ $0.98, I^{2}=0 \%$; Figure 3 ). Therefore, fixed-effect model was applied for RR. Meta-analysis showed a significant difference in intrauterine fetal distress between the two groups $(\mathrm{RR}=$ $0.26,95 \% \mathrm{Cl}: 0.09-0.70, p<0.01)$. The use of Compound Danshen injection alone or in combination with Western medicine significantly reduced the risk of intrauterine fetal distress in the $\mathrm{PIH}$ patients.

\section{Caesarean section}

Statistical analysis of four (04) studies that discussed caesarean section showed absence of heterogeneity among the various studies ( $p=$ $0.14,1^{2}=45 \%$; Figure 4). Thus, the fixed-effect model was used for calculation of RR. Statistical significance was found in meta-analysis of caesarean section between the two groups (RR $=0.72,95 \% \mathrm{Cl}: 0.58$ - 0.90), $p<0.01)$. Administration of Compound Danshen injection alone or in combination with western medicine significantly reduced the risk of caesarean section in patients with $\mathrm{PIH}$.

\section{Postpartum hemorrhage}

Five (05) studies reported postpartum hemorrhage, with significant heterogeneity among them $\left(p=0.0002, \mathrm{I}^{2}=82 \%\right.$; Figure 5$)$. Thus, the random-effect model was used for RR. Meta-analysis did not demonstrate significant difference between the two groups $(R R=0.49$, $95 \% \mathrm{Cl}=0.09-2.76, p>0.05)$. 


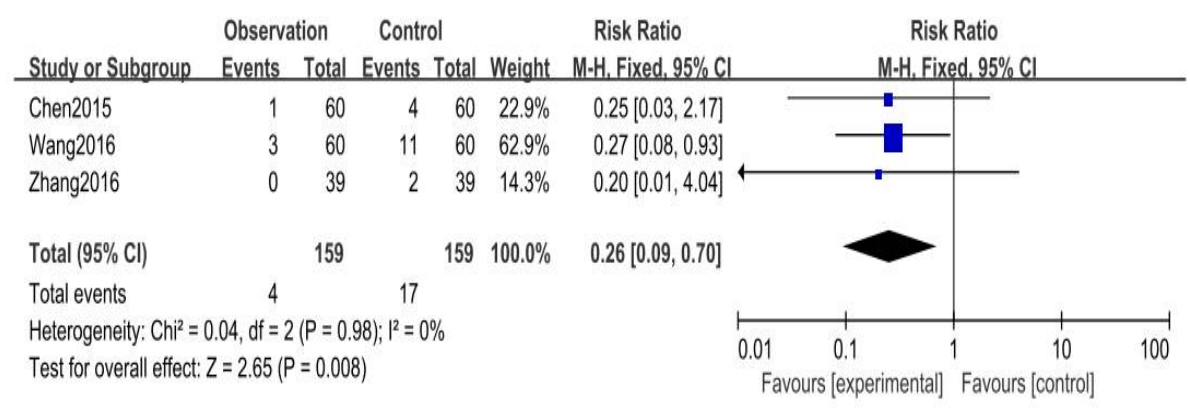

Figure 3: Forest plot of meta-analysis of intrauterine fetal distress

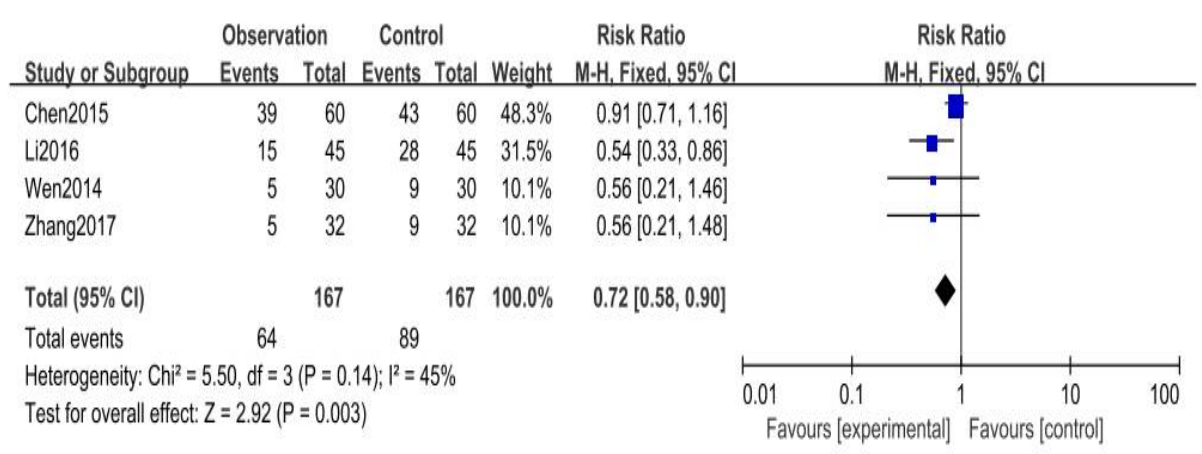

Figure 4: Forest plot of meta-analysis of caesarean section

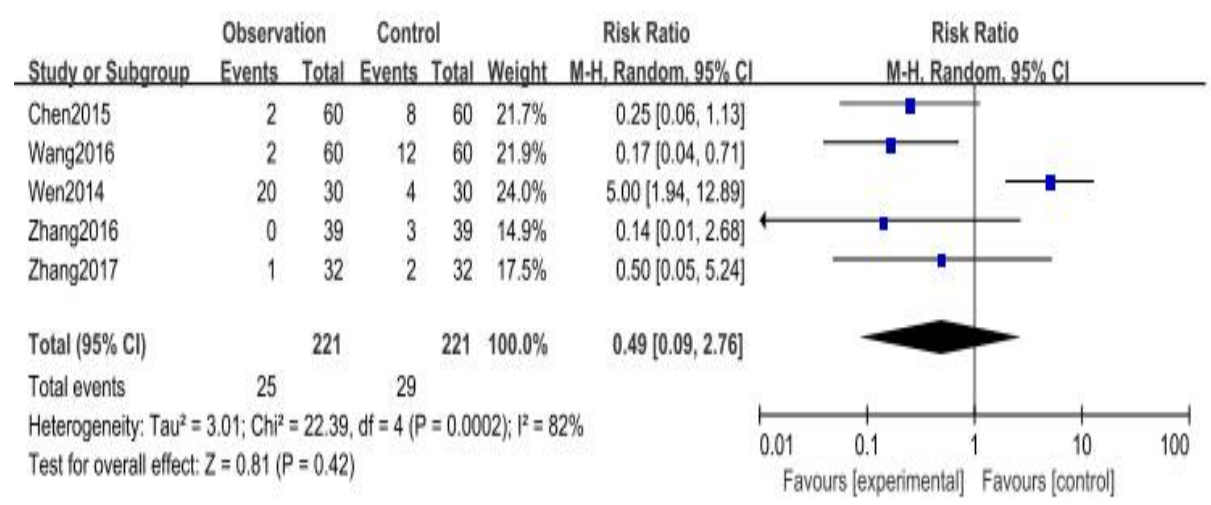

Figure 5: Forest plot of meta-analysis of postpartum hemorrhage

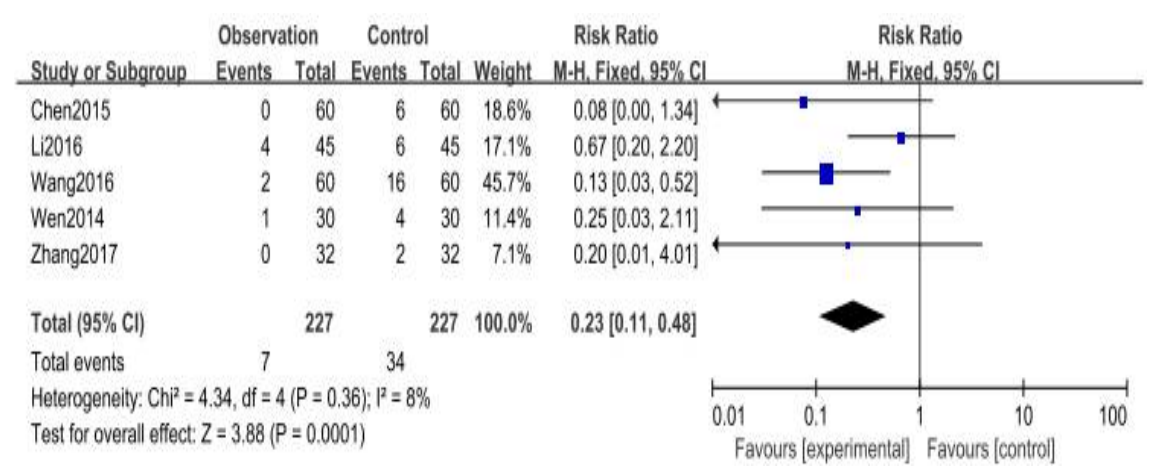

Figure 6: Forest plot of meta-analysis of neonatal asphyxia

\section{Neonatal asphyxia}

Five (05) studies discussed neonatal asphyxia. Heterogeneity test showed no significant heterogeneity among the studies $\left(p=0.36, \mathrm{I}^{2}=8\right.$ $\%$; Figure 6 ). Thus, the fixed-effect model was used for calculating RR. Meta-analysis showed statistically significant difference in neonatal asphyxia between the two groups ( $R R=0.23$, 95 $\% \mathrm{Cl}: 0.11-0.48, p<0.0001)$. It was revealed that Compound Danshen injection alone or in 
combination with Western medicine significantly reduced the risk of neonatal asphyxia.

\section{Publication bias}

As seen from the funnel plot analysis (Figure 7), evaluation of the funnel plot symmetry with total effectiveness showed that there was no significant publication bias in the various publications. The selected studies were representative.

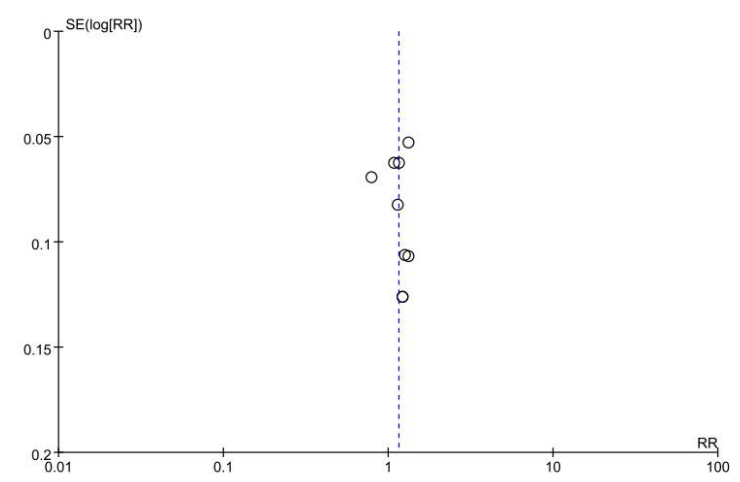

Figure 7: Begg's Funnel plot for publication bias test. Each circle denotes an independent study for the indicated association. Log $[R R]=$ natural logarithm of RR. Horizontal line stands for mean effect size

\section{Adverse reactions}

There were reports of adverse reactions in three (03) studies. Adverse reactions were lower and lighter in the observation groups than in the control groups. Most of the adverse reactions were mild diarrhea, skin eruption, and a few cases of leucopenia.

\section{DISCUSSION}

Pregnancy-induced hypertension (PIH) seriously affects the safety and quality of life of mothers and infants [19]. A large-scale study found that the probability of maternal death in mothers diagnosed with PIH after pregnancy was significantly higher than that in normal pregnant women [20]. In addition, PIH is often combined with a number of other disease conditions such as pulmonary edema and liver dysfunction, which also increase the risk of pregnancy-related death $[21,22]$. With incidence of $9 \%$ and mortality of $3.3 / 100,000$, PIH exerts a great burden on medical and social expenditures in China [23].

Many studies on the etiology and pathogenesis of $\mathrm{PIH}$ have suggested the involvement of systemic arteriole spasm. The main therapeutic measures for $\mathrm{PIH}$ are symptomatic strategies such as antispasmodic and antihypertensive treatments, as well as dilatation, diuresis, and timely termination of pregnancy. Western medicine is often preferred to magnesium sulfate. However, the effective concentration of magnesium sulfate is close to its toxic dose which can easily cause maternal and infant magnesium poisoning. Thus, there is need to actively seek ways of reducing the dose of the drug so as to avoid side effects while improving its effectiveness. Traditional Chinese medicine has shown that PIH is associated with blood stasis. Due to yang deficiency of kidney and poor blood stasis, the hemo-rheological changes induced by systemic arteriole spasm in patients with PIH provide objective indicators of blood stasis.

Compound Danshen injection is a pure Chinese medicine preparation extracted from the dried roots of Salvia miltiorrhiza and dalbergiae. Its main components are tanshinone II-A, sodium sulfonate and danshensu. Salvia miltiorrhiza is used for enhancing blood circulation by removing blood stasis, and for dilating blood vessels, removing free radicals, and blocking calcium channels. Dalbergiae is effective in removing blood stasis and relieving swelling and pain.

In this study, comparison of the effectiveness and safety between Compound danshen injection and conventional Western medicine for $\mathrm{PIH}$ treatment revealed that Compound danshen injection resulted in better total effectiveness and quality of life of PIH patients and infants. Due to limited number of studies used, the analysis did not show a significant difference in risk of fetal heart abnormalities and postpartum hemorrhage between compound Danshen injection and conventional Western medicine. Treatment with combination of Compound Danshen injection and Western medicine (magnesium sulfate, nifedipine and others) reduced the dose of magnesium sulfate, thereby avoiding the occurrence of adverse reactions such as magnesium sulfate poisoning.

\section{Study limitations}

The present study has several limitations, including the meta-analysis. Although the results obtained revealed that Compound Danshen injection was superior to conventional Western medicine in PIH treatment, $\mathrm{PIH}$ is a complex disease resulting from the interaction of many factors. Thus, these results require verification using a large-sample, multi-center randomized and controlled trial study in the future. 


\section{CONCLUSION}

The results obtained in this study suggest that the effectiveness of PIH treatment with Compound Danshen injection alone or in combination with Western medication is superior to that of conventional western medical treatment. More importantly, the combination treatment improves the safety of pregnant women, fetuses and newborns. Thus, it is worthy of clinical application.

\section{DECLARATIONS}

\section{Conflict of Interest}

No conflict of interest associated with this work.

\section{Contribution of Authors}

The authors declare that this work was done by the authors named in this article and all liabilities pertaining to claims relating to the content of this article will be borne by them.

\section{REFERENCES}

1. Ren $X Y$, Tan $L H$, Hao $L H$. The effect of compound danshen on the renal function with severe pregnancyinduced hypertension. Chin Matern Child Health 2009; 19(8):46-47.

2. Ma DF, Yang Y. Effects of Compound Danshen Injection on Coagulation and Fibrinolytic System Index in Patients with Pregnancy-induced Hypertension Syndrome. Shizhen Guoyi Med Drugs 2008; 19(8): 2020-2021.

3. Guo CY. Compound Danshen on serum uric acid levels in patients with pregnancy-induced hypertension syndrome. Modern J Integr Tradit Chin Western Med 2009 ; 18(11): 1247, 1299.

4. Hao DJ, Wei JP, Zhang LN. Clinical comparison of pregnancy induced hypertension syndrome by magnesium Sulfate and compound danshen injection. Modern prev med 2009; 36(14): 2785-2786.

5. Chu $H$. Effects of magnesium sulfate and compound danshen injection on plasma nitric oxide and endothelin levels in the patients with pregnancy induced hypertension. Chin sex sci 2012; 21(12): 47-49.

6. Zheng AR. Clinical Observation of Magnesium Sulfate Combined with Compound Danshen Injection in Treating Pregnancy-induced Hypertension. Strait Pharmaceut 2013; 25(3): 71-72.

7. Jia XL. Clinical observation of drug treatment of hypertensive disorders in pregnancy. China Health Ind 2013; 05(C): 24, 26.

8. Wang D, Wang QJ. Clinical observation of Pregnancyinduced Hypertension by Compound Danshen Injection Chin Prac Med 2014; 7(9): 162-163.
9. Wen SZ. Clinical observation of Pregnancy-induced Hypertension by Compound Danshen Injection. Tradit Chin med 2014; 32(12): 285-286.

10. Zhang JL. Clinical Observation of combination of Chinese and Western medicine in Treating Pregnancy-induced Hypertension. Cardiovasc Dis J of integr tradit Chin Western Med, 2015; 3(9): 67, 69.

11. Chen TT. The Effect of compound Danshen injection renal adjuvant therapy on maternal and infant pregnancy Outcome in Pregnancy-induced Hypertension. China Prac Med- 2015; 10(22): 139-141.

12. Qiu DH. Effect of compound danshen injection on expression of GHS-RmRNA and Ghrelin in patients with pregnancy induced hypertension. Clin Drug Appl 2016; 24(6): 38-40.

13. Li KH, Chen HY, Fu J, Wang $X, W u H X$. The Effect of FuFang Injection Combined with Magnesium Sulfate on Pregnancy Outcome in Patients with Hypertension and ET syndrome of NO. World Chin Med 2016; 11(4): 636638.

14. Wang LJ, Zhang TL, Liu WJ. Clinical Observation of Compound Danshen Injection, vitamin $E$ and calcium in Treating Pregnancy-induced Hypertension. Hebei Med J 2016; 38(27): 3588-3591.

15. Ding DY. Clinical Observation of Magnesium Sulfate Combined with Compound Danshen Injection in Treating Pregnancy-induced Hypertension. Chin J Prac Gynecol Obstetr 2016; 5(3): 28-29.

16. Zhang ZX. Clinical Observation of Compound Danshen Injection Combined with nifedipine in Treating Pregnancy-induced Hypertension. Gansu Sci Technol 2011; 32(14): 123-124.

17. Zhao LM. The Effect of Compound Danshen Injection on plasma $D$ two dimer level in Pregnancy-induced Hypertension. The $J$ of Med THEORY PRACT, 2017; 30(11): 1654-1656.

18. Zhang RD. Clinical Observation of Compound Danshen Injection in Treating Pregnancy-induced Hypertension. Shenzhen J of Integr Tradit Chin Western Med 2015; 27(17): 39-40.

19. Luizon MR, Palei ACT, Cavalli RC, Sandrim VC. Pharmacogenetics in the treatment of pre-eclampsia: current findings, challenges and perspectives. Pharmacogenomics 2017; 18(6): 571-583.

20. Nobumoto E, Masuyama H, Maki J, Eguchi T, Tamada S, Mitsui T, Eto E, Hayata K, Hiramatsu Y. Comparison of Kidney Function between Gestational Hypertension and Preeclampsia. Acta Med Okayama 2017; 71(2): 161 169.

21. Draganovic D, Lucic N, Jojic D. Oxidative Stress Marker and Pregnancy Induced Hypertension. Med Arch 2016; 70(6): 437-440.

22. Tandon V, Hiwale S, Amle D. Assessment of Serum Vascular Endothelial Growth Factor Levels in Pregnancy-Induced Hypertension Patients. J Pregnancy 2017; 1-5.

23. Liu YQ, Tan GL, Shang CM. The ICU Is Becoming a Main Battlefield for Severe Maternal Rescue in China:

Trop J Pharm Res, January 2019; 18(1): 200 
Wang et al 